\title{
Application of genomics to identify therapeutic targets in recurrent pediatric papillary thyroid carcinoma
}

\author{
Rebecca Ronsley, ${ }^{1}$ S. Rod Rassekh, ${ }^{2,3}$ Yaoqing Shen, ${ }^{4}$ Anna F. Lee, ${ }^{5}$ \\ Colleen Jantzen, ${ }^{3}$ Jessica Halparin, ${ }^{3}$ Catherine Albert, ${ }^{6}$ Douglas S. Hawkins, ${ }^{6}$ \\ Shazhan Amed, ${ }^{1}$ Ralph Rothstein, ${ }^{1}$ Andrew J. Mungall, ${ }^{4}$ David Dix, ${ }^{3}$ \\ Geoffrey Blair, ${ }^{7}$ Helen Nadel, ${ }^{8}$ Steven J.M. Jones, ${ }^{4}$ Janessa Laskin, ${ }^{9,11}$ Marco
} A. Marra, ${ }^{4,10,11}$ and Rebecca J. Deyell ${ }^{2,3,11}$

\begin{abstract}
${ }^{1}$ Division of Endocrinology and Metabolism, University of British Columbia, Vancouver, British Columbia V6T 1Z4, Canada; ${ }^{2}$ BC Children's Hospital Research Institute, Vancouver, British Columbia V5Z 4H4, Canada; ${ }^{3}$ Division of Oncology and Hematology, Department of Pediatrics, University of British Columbia, Vancouver, British Columbia V6T 1Z4, Canada; ${ }^{4}$ Canada's Michael Smith Genome Science Centre, British Columbia Cancer, Vancouver, British Columbia V5Z 4S6, Canada; ${ }^{5}$ Department of Pathology and Laboratory Medicine, University of British Columbia, Vancouver, British Columbia V6T 1Z4, Canada; 'Division of Hematology/ Oncology, Department of Pediatrics, Seattle Children's Hospital, University of Washington, Fred Hutchinson Cancer Research Center, Seattle, Washington 98105, USA; ${ }^{7}$ Department of Surgery, University of British Columbia, Vancouver, British Columbia V6T 1Z4, Canada; ${ }^{8}$ Department of Radiology, University of British Columbia, Vancouver, British Columbia V6T 1Z4, Canada; ${ }^{9}$ British Columbia Cancer, Vancouver, British Columbia V5Z 4S6, Canada; ${ }^{10}$ Department of Medical Genetics, University of British Columbia, Vancouver, British Columbia V6T 1Z4, Canada
\end{abstract}

Corresponding author: rdeyell@cw.bc.ca

(C) 2018 Ronsley et al. This article is distributed under the terms of the Creative Commons Attribution-NonCommercial License, which permits reuse and redistribution, except for commercial purposes, provided that the original author and source are credited.

Ontology terms: metastatic angiosarcoma; papillary thyroid carcinoma

Published by Cold Spring Harbor Laboratory Press

doi: $10.1101 /$ mcs.a002568
Abstract Children with papillary thyroid carcinoma (PTC) may relapse despite response to radioactive iodine (RAI). Two children with multiply relapsed PTC underwent whole-genome and transcriptome sequencing. A TPM3-NTRK1 fusion was identified in one tumor, with outlier NTRK1 expression compared to the TCGA thyroid cancer compendium and to Illumina BodyMap normal thyroid. This patient demonstrated resolution of multiple pulmonary nodules without toxicity on oral TRK inhibitor therapy. A RET fusion was identified in the second tumor, another potentially actionable finding. Identification of oncogenic drivers in recurrent pediatric PTC may facilitate targeted therapy while avoiding repeated RAI.

\section{INTRODUCTION}

Papillary thyroid carcinoma (PTC) is the most commonly diagnosed thyroid cancer in childhood (Greco et al. 2010). Compared to adults, children are more likely to present with advanced local disease as well as widespread metastases. The treatment of locally invasive pediatric PTC is complete surgical excision followed by radioactive iodine (RAl) ablation (Parisi et al. 2016). Although overall survival is excellent, the recurrence rate remains high at $40 \%$ and some children have multiple relapses, requiring additional surgeries and repeated RAl (Parisi et al. 2016). There are significant acute and late effects associated with RAI (Rubino et al. 2003; Florenzano et al. 2016), which are increased in patients who receive higher cumulative doses (Rubino et al. 2003; Fard-Esfahani et al. 2014).

\footnotetext{
${ }^{11}$ Co-senior authors
} 
Activating point mutations and oncogenic fusions in NTRK, RET, RAS, and BRAF have been identified in PTC (Beimfohr et al. 1999; Xing et al. 2013; Khotskaya et al. 2017). Compared with adults, pediatric PTC are more likely to have fusion proto-oncogenes, which are associated with more aggressive and disseminated disease (Prasad et al. 2016). NTRK1/3 fusion oncogenes (located on Chromosome 1) are rare in adult PTC (Fig. 1A; Nikiforov and Nikiforova 2011; The Cancer Genome Atlas Research Network 2014) but described in up to $26 \%$ of pediatric PTC (Prasad et al. 2016). RET gain of function mutations (located on Chromosome 10) (Fig. 1B) have been described in many endocrine cancers, and more

A
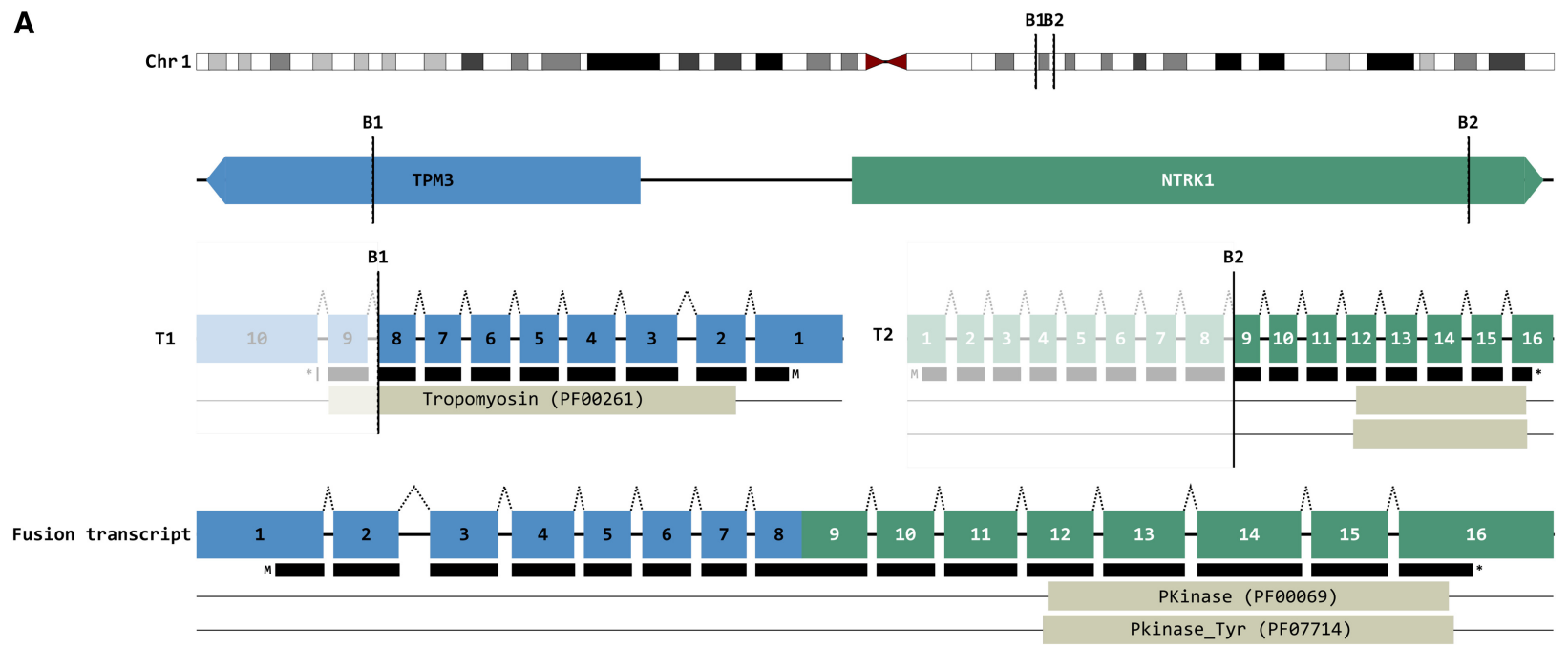

B
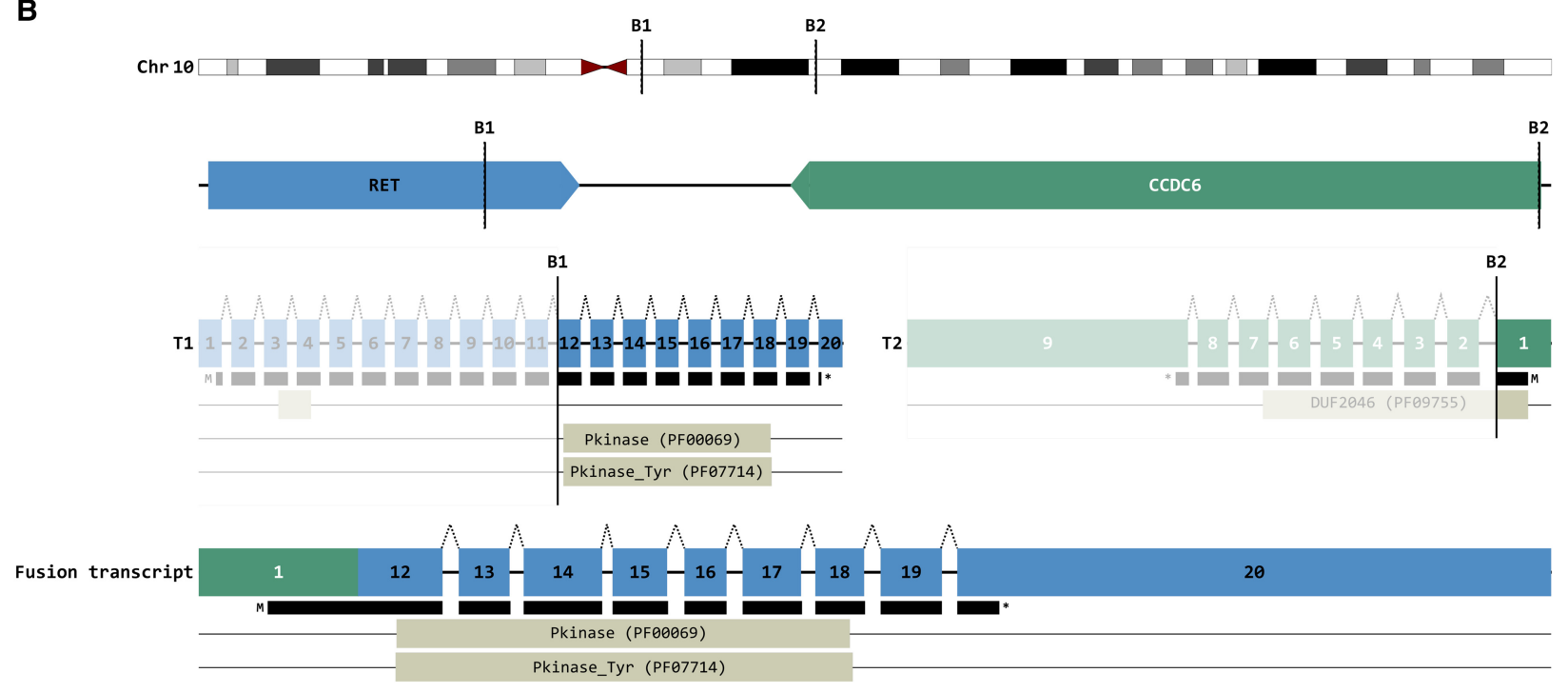

Figure 1. TPM3-NTRK1 and RET-CCDC6 oncogenic fusions with exon numbers and protein domains. (A) TPM3-NTRK1 fusion. Chr 1, Chromosome 1; B1, breakpoint that falls into TPM3; B2, breakpoint that falls into NTRK1; T1, transcript of TPM3 (ENST00000368530); T2, transcript of NTRK1 (ENST00000368196). The numbers on T1 and T2 indicate exons. Pfam domains and accession IDs are labeled beneath the transcripts. (B) RET-CCDC6 fusion. Chr 10, Chromosome 10; B1, breakpoint that falls into RET; B2, breakpoint that falls into CCDC6; T1, transcript of RET (ENST00000355710); T2, transcript of CCDC6 (ENST00000263102). 
COLD SPRING HARBOR Molecular Case Studies
Therapeutic targets in recurrent pediatric papillary thyroid carcinoma

than 10 activating RET fusions have been identified in thyroid cancers (Menicali et al. 2012; Accardo et al. 2017).

The identification of recurrent oncogenic drivers in aggressive PTC presents the opportunity for targeted therapy. An adult phase I study in NTRK fusion driven malignancies and advanced disease have demonstrated partial responses in the first three evaluable patients with minimal toxicity (American Association for Cancer Research 2016). An ongoing pediatric phase I/II trial of a pan-TRK inhibitor, larotrectinib, has demonstrated tumor responses across histologic cancer types in TRK-fusion driven cancers (NCT02637687) (Laetsch et al. 2017).

There is also evidence to support a role for oral RET inhibitors in thyroid cancer. In a phase 1 study in adults with RAl-refractory differentiated thyroid cancer, $53 \%$ had a response by RECIST to cabozantinib, an oral multikinase inhibitor for RET, MET, and VEGFR2 (Cabanillas et al. 2014). Cabozantinib is FDA-approved in adults with metastatic medullary thyroid carcinoma and renal cell carcinoma (Food and Drug Administration 2016). A pediatric phase I study was recently completed, with a phase II expansion currently accruing patients with refractory solid tumors, including medullary thyroid carcinoma (NCT01709435 and NCT02867592) (Akshintala and Children's Oncology Group 2017).

We describe whole-genome and transcriptome sequencing in two children with RAI-refractory PTC and document radiographic improvement in a patient with an oncogenic TPM3NTRK 1 fusion following TRK inhibitor therapy.

\section{RESULTS}

\section{Case 1}

A previously healthy 12 -yr-old boy presented with a painless right neck mass without constitutional symptoms, no family history of cancer, no radiation exposure, endocrinopathies or underlying syndromes. An ultrasound showed a large, heterogeneous solid mass arising from the right thyroid lobe, extending into the isthmus with localized lymphadenopathy. A total thyroidectomy confirmed PTC involving the right lobe and microscopic involvement of the isthmus and left lobe. Surgical margins were positive with multiple involved lymph nodes. Postoperatively, he received $100 \mathrm{mCi}$ of $\mathrm{I}^{131} \mathrm{RAI}$ and was maintained euthyroid with thyroid hormone replacement.

One year later, his thyroglobulin levels rose and imaging confirmed relapse within the neck, thyroid bed and paratracheal lymph nodes, along with new bilateral pulmonary nodules. He underwent cervical debulking surgery and received an additional $150 \mathrm{mCi}$ of $\mathrm{I}^{131}$ RAI. Follow-up $I^{131}$ imaging demonstrated progression of multiple bilateral, small pulmonary nodules with active uptake in several neck and thyroid bed lymph nodes. Repeat neck and chest CT two months following repeat RAI revealed progression of innumerable pulmonary metastatic nodules. The patient was enrolled in the POG project, and sections from his surgical resection were sent for WGS and transcriptome sequencing. From the sequencing data we identified four somatic nonsynonymous mutations and one indel, none of which have known clinical significance (Table 1). Analysis of sequencing data results revealed a somatic TPM3::NTRK1(e8:e9) fusion (Fig. 1A), reported previously in PTC. The same fusion was validated by PCR and was also identified on archival tissue from diagnosis. Furthermore, there was very high NTRK1 expression in the tumor compared to a normal thyroid tissue from Illumina Bodymap, and to the TCGA thyroid cancer samples (THCA) (Fig. 2A). In addition to NTRK1, other genes encoding receptor tyrosine kinases (RTKs) including PDGFRB and EGFR also show high percentile compared to THCA. In the hedgehog pathway, GLI1, GLI2, and GLI3 all show high level of expression (95th-98th percentile compared to THCA). 
Table 1. Somatic nonsynonymous small mutations and indels in Patient 1

\begin{tabular}{|c|c|c|c|c|c|c|c|c|c|}
\hline Gene & Chromosome & $\begin{array}{l}\text { HGVS DNA } \\
\text { reference }\end{array}$ & $\begin{array}{l}\text { HGVS protein } \\
\text { reference }\end{array}$ & $\begin{array}{l}\text { Variant } \\
\text { type }\end{array}$ & $\begin{array}{l}\text { Predicted } \\
\text { effect }\end{array}$ & dbSNP & Genotype & $\begin{array}{c}\text { VAF in } \\
\text { tumor } \\
\text { genome }^{a}\end{array}$ & Note \\
\hline MUC5B & 11 & $\begin{array}{r}\text { NM_002458.2: } \\
\text { c. } 12272 C>G\end{array}$ & $\begin{array}{l}\text { NP_002449.2: } \\
\text { p.Thr4091Arg }\end{array}$ & Substitution & Missense & rs199736618 & het & $17 \%$ & \\
\hline$B A Z 2 B$ & 2 & $\begin{array}{l}\text { NM_013450.2: } \\
\text { c.197C }>T\end{array}$ & $\begin{array}{l}\text { NP_038478.2: } \\
\text { p.Ser66Leu }\end{array}$ & Substitution & Missense & & het & $26 \%$ & \\
\hline CRIPAK & 4 & $\begin{array}{l}\text { NM_175918.3: } \\
\text { c. } 738 \mathrm{G}>\mathrm{A}\end{array}$ & $\begin{array}{r}\text { NP_787114.2: } \\
\text { p.Met246lle }\end{array}$ & Substitution & Missense & rs76723438 & het & $15 \%$ & \\
\hline$D S P P$ & 4 & $\begin{array}{c}\text { NM_014208.3: } \\
\text { c.3139G >A }\end{array}$ & $\begin{array}{l}\text { NP_055023.2: } \\
\text { p.Asp1047Asn }\end{array}$ & Substitution & Missense & & het & $15 \%$ & $\begin{array}{l}\text { May be a } \\
\text { sequencing } \\
\text { artifact }\end{array}$ \\
\hline$L R R C 40$ & 1 & $\begin{array}{l}\text { NM_017768.4: } \\
\text { c.687_691del }\end{array}$ & $\begin{array}{l}\text { NP_060238.3: } \\
\text { p.Asn230LeufsTer7 }\end{array}$ & Deletion & Frameshift & & het & $22 \%$ & \\
\hline
\end{tabular}

HGVS, Human Genome Variation Society; VAF, variant allele frequency; het, heterozygous.

aith $58 \%$ tumor content in the sequenced sample.

The patient was enrolled on a pediatric phase I/II study (NCT02637687) evaluating an oral, selective pan-TRK inhibitor, larotrectinib (Laetsch et al. 2017). Following two cycles of TRK inhibitor therapy, repeat CT chest demonstrated an overall reduction in number of pulmonary nodules (Fig. 3A,B). Subsequent CT chest after four and six cycles revealed further interval improvement, with excellent functional status (Lansky 100) without any

A

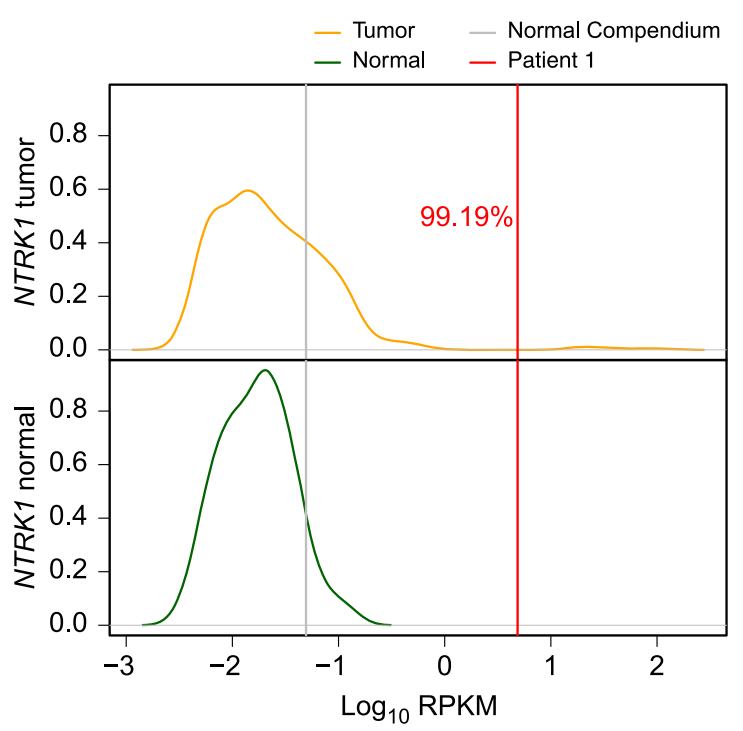

B

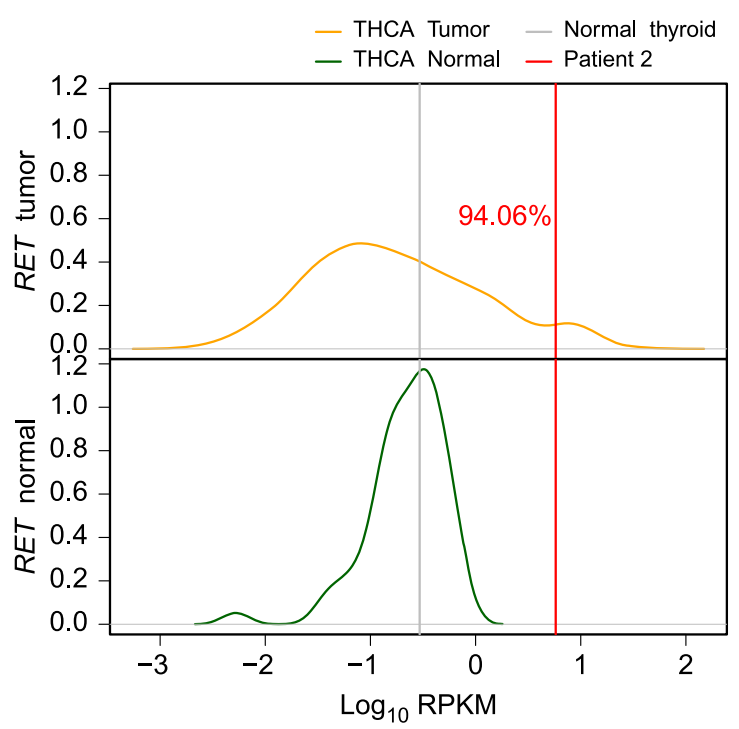

Figure 2. (A) High NTRK1 RNA expression in Patient 1 compared to 513 tumor samples from TCGA thyroid (THCA) cohort and 59 matched normal samples. The distributions of NTRK1 expression in the tumor and normal groups are shown as density curve. The gray line indicates NTRK1 expression in a normal thyroid sample from Illumina body map. (B) High RET RNA expression in Patient 2 compared to 513 tumor samples from TCGA thyroid (THCA) cohort and 59 matched normal samples. The distributions of RET expression in the tumor and normal groups are shown as density curve. The gray line indicates RET expression in a normal thyroid sample from Illumina body map. 
A

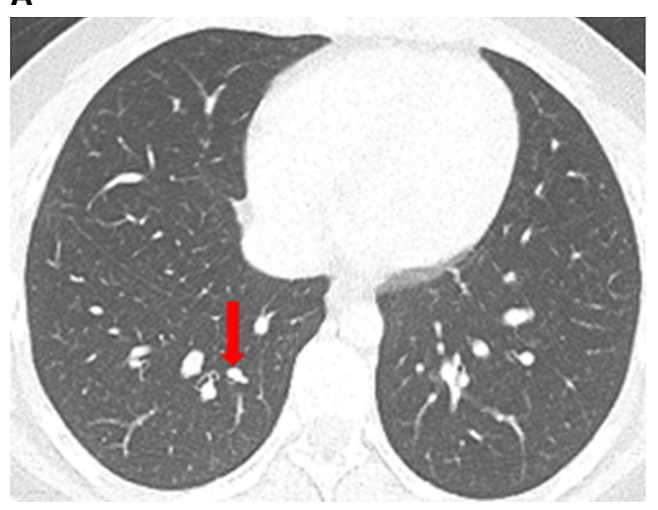

B

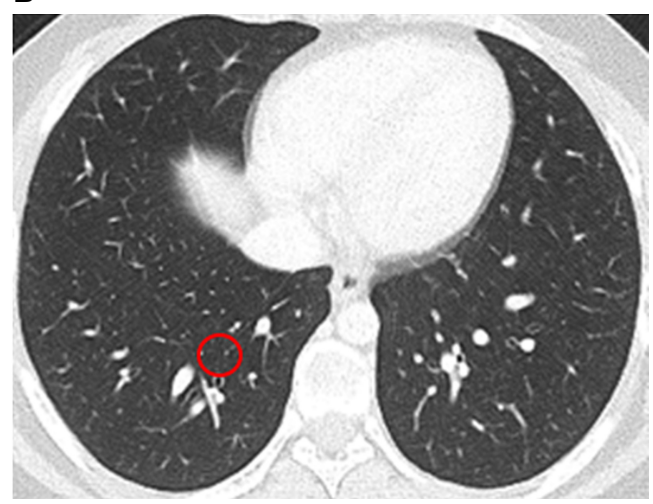

Figure 3. Pre- and posttherapy CT chest images for Case 1. (A) Axial CT chest image demonstrating a large (6$\mathrm{mm}$ ) pulmonary nodule (red arrow) prior to oral TRK inhibitor therapy initiation. At baseline, the patient had progressive innumerable, small pulmonary nodules demonstrated throughout the lungs bilaterally. (B) Coregistered axial CT image following four cycles of oral TRK inhibitor therapy demonstrating resolution of the prior pulmonary nodule (red circle) documented at baseline.

therapy-related toxicity. Currently, the patient is 10 months post initiation of therapy with almost complete resolution of prior innumerable pulmonary nodules, and has excellent functional status.

\section{Case 2}

A previously healthy 13 -yr-old girl presented with a three-year history of an enlarging rightsided goiter and a neck ultrasound demonstrating enlarged, calcified cervical lymph nodes. Fine needle aspiration was suspicious for PTC and the patient underwent a total thyroidectomy with right radical neck dissection. Pathology confirmed PTC involving both thyroid lobes, the isthmus as well as extensive involvement of regional lymph nodes. The patient underwent I $\mathrm{I}^{131} \mathrm{RAI}$ therapy of $100 \mathrm{mCi}$. Six months postoperatively, neck ultrasound demonstrated enlarging cervical adenopathy and a cervical lymph node excisional biopsy confirmed locally recurrent PTC in two nodes. Thyrogen stimulated positron emission tomography-computed tomography (PET-CT) demonstrated marked 18-fluoro-deoxy-glucose (FDG) avidity in the thyroid bed and right cervical chain, with no evidence of distant disease. The patient underwent a third surgery with a lymph node biopsy and a modified left radical neck dissection. Resected lymph nodes were again positive for PTC and the patient received an additional I ${ }^{131}$ RAl treatment.

The patient's tumor at relapse underwent whole-genome and transcriptome sequencing in POG. We identified 23 somatic nonsynonymous small mutations in the genome, none of which have known clinical significance (Table 2). We also found a CCDC6::RET (e1:e12) fusion that is frequently reported in PTC (Fig. 1B; Santoro and Carlomagno 2013). In the fused protein, RET loses the extracellular part (for ligand binding), but the kinase domain is intact. Such fusion drives the expression and ligand-independent activation of the fused protein (Nikiforov and Nikiforova 2011). In this case, RET shows high expression when compared to TCGA thyroid cancer, and normal thyroid tissue from Illumina bodymap (Fig. 2B). As with Patient 1, PDGFRB, EGFR, GLI1, GLI2, and GLI3 all showed high percentile compared to TCGA THCA.

Several receptor tyrosine kinase inhibitors such as cabozantinib, regorafenib, vandetanib, sorafenib, and sunitinib show activity against RET. Preclinical study on PTC cell-line with RET fusion showed that XL184 (cabozantinib) is more potent in inhibiting cell growth, 
Table 2. Somatic nonsynonymous small mutations in Patient 2

\begin{tabular}{|c|c|c|c|c|c|c|c|c|}
\hline Gene & Chromosome & $\begin{array}{l}\text { HGVS DNA } \\
\text { reference }\end{array}$ & $\begin{array}{l}\text { HGVS protein } \\
\text { reference }\end{array}$ & $\begin{array}{l}\text { Variant } \\
\text { type }\end{array}$ & $\begin{array}{l}\text { Predicted } \\
\text { effect }\end{array}$ & $\mathrm{dbSNP}$ & Genotype & $\begin{array}{l}\text { VAF in } \\
\text { tumor } \\
\text { genome }^{a}\end{array}$ \\
\hline $\mathrm{TCHH}$ & 1 & $\begin{array}{c}\text { NM_007113.3: } \\
\text { c. } 1030 \mathrm{G}>\mathrm{C}\end{array}$ & $\begin{array}{l}\text { NP_009044.2: } \\
\text { p.Glu344GIn }\end{array}$ & Substitution & Missense & rs201434724 & het & $9 \%$ \\
\hline FCGR2A & 1 & $\begin{array}{l}\text { NM_021642.3: } \\
\text { c.818T >C }\end{array}$ & $\begin{array}{l}\text { NP_067674.2: } \\
\text { p.Leu273Pro }\end{array}$ & Substitution & Missense & & het & $14 \%$ \\
\hline CD163L1 & 12 & $\begin{array}{c}\text { NM_174941.5: } \\
\text { c. } 2332 \mathrm{G}>\mathrm{T}\end{array}$ & $\begin{array}{l}\text { NP_777601.2: } \\
\text { p.Ala778Ser }\end{array}$ & Substitution & Missense & & het & $10 \%$ \\
\hline PRB4 & 12 & $\begin{array}{c}\text { NM_002723.5: } \\
\text { c. } 347 C>A\end{array}$ & $\begin{array}{l}\text { NP_002714.2: } \\
\text { p.Pro116His }\end{array}$ & Substitution & Missense & rs59189129 & het & $14 \%$ \\
\hline $\mathrm{DACH} 1$ & 13 & $\begin{array}{c}\text { NM_004392.6: } \\
\text { c. } 235 \mathrm{G}>\mathrm{A}\end{array}$ & $\begin{array}{c}\text { NP_004383.4: } \\
\text { p.Gly79Ser }\end{array}$ & Substitution & Missense & rs200162861 & het & $20 \%$ \\
\hline PAPLN & 14 & $\begin{array}{c}\text { NM_173462.3: } \\
\text { c.2041C >T }\end{array}$ & $\begin{array}{l}\text { NP_775733.3: } \\
\text { p.Gln681Ter }\end{array}$ & Substitution & Stop_gained & & het & $17 \%$ \\
\hline TPSB2 & 16 & $\begin{array}{l}\text { NM_024164.5: } \\
\text { c. } 253 \mathrm{G}>\mathrm{A}\end{array}$ & $\begin{array}{c}\text { NP_077078.5: } \\
\text { p.Ala85Thr }\end{array}$ & Substitution & Missense & rs202041848 & het & $17 \%$ \\
\hline EXOC3L1 & 16 & $\begin{array}{l}\text { NM_178516.3: } \\
\text { c. } 41 C>G\end{array}$ & $\begin{array}{c}\text { NP_848611.2: } \\
\text { p.Ser14Cys }\end{array}$ & Substitution & Missense & & het & $17 \%$ \\
\hline ZNF844 & 19 & $\begin{array}{l}\text { NM_001136501.2: } \\
\text { c. } 1459 T>C\end{array}$ & $\begin{array}{c}\text { NP_001129973.1: } \\
\text { p.Phe487Leu }\end{array}$ & Substitution & Missense & & het & $13 \%$ \\
\hline ZNF430 & 19 & $\begin{array}{c}\text { NM_025189.3: } \\
\text { c.869G >A }\end{array}$ & $\begin{array}{l}\text { NP_079465.3: } \\
\text { p.Arg290Lys }\end{array}$ & Substitution & Missense & & het & $9 \%$ \\
\hline TRIOBP & 22 & $\begin{array}{l}\text { NM_001039141.2: } \\
\text { c.2072T>C }\end{array}$ & $\begin{array}{l}\text { NP_001034230.1: } \\
\text { p.lle691Thr }\end{array}$ & Substitution & Missense & & het & $20 \%$ \\
\hline ZNF852 & 3 & $\begin{array}{l}\text { XM_001717544.3: } \\
\text { c. } 1477 G>A\end{array}$ & $\begin{array}{l}\text { XP_001717596.3: } \\
\text { p.Glu493Lys }\end{array}$ & Substitution & $\begin{array}{l}\text { Missense + } \\
\text { splice_region }\end{array}$ & rs113868900 & hom & $46 \%$ \\
\hline MUC4 & 3 & $\begin{array}{r}\text { NM_018406.6: } \\
\text { c.11633T>C }\end{array}$ & $\begin{array}{l}\text { NP_060876.5: } \\
\text { p.Leu3878Pro }\end{array}$ & Substitution & Missense & & het & $20 \%$ \\
\hline ART3 & 4 & $\begin{array}{c}\text { NM_001179.5: } \\
\text { c.376C>T }\end{array}$ & $\begin{array}{l}\text { NP_001170.2: } \\
\text { p.Arg126Ter }\end{array}$ & Substitution & Stop_gained & & het & $20 \%$ \\
\hline PRDM9 & 5 & $\begin{array}{c}\text { NM_020227.3: } \\
\text { c. } 1696 C>G\end{array}$ & $\begin{array}{l}\text { NP_064612.2: } \\
\text { p.His566Asp }\end{array}$ & Substitution & Missense & & het & $12 \%$ \\
\hline MUC21 & 6 & $\begin{array}{l}\text { NM_001010909.3: } \\
\text { c.743C>T }\end{array}$ & $\begin{array}{l}\text { NP_001010909.2: } \\
\text { p.Pro248Leu }\end{array}$ & Substitution & Missense & rs41288651 & het & $16 \%$ \\
\hline MUC22 & 6 & $\begin{array}{l}\text { NM_001198815.1: } \\
\text { c.2969A>C }\end{array}$ & $\begin{array}{l}\text { NP_001185744.1: } \\
\text { p.Glu990Ala }\end{array}$ & Substitution & Missense & & het & $9 \%$ \\
\hline ZAN & 7 & $\begin{array}{c}\text { NM_003386.1: } \\
\text { c. } 2140 C>A\end{array}$ & $\begin{array}{l}\text { NP_003377.1: } \\
\text { p.Pro714Thr }\end{array}$ & Substitution & Missense & & het & $10 \%$ \\
\hline MUC12 & 7 & $\begin{array}{c}\text { NM_001164462.1: } \\
\text { c.5495C>T }\end{array}$ & $\begin{array}{l}\text { NP_001157934.1: } \\
\text { p.Thr1832lle }\end{array}$ & Substitution & Missense & & het & $14 \%$ \\
\hline MUC17 & 7 & $\begin{array}{l}\text { NM_001040105.1: } \\
\text { c.8225G>C }\end{array}$ & $\begin{array}{c}\text { NP_001035194.1: } \\
\text { p.Arg2742Pro }\end{array}$ & Substitution & Missense & rs141347629 & het & $11 \%$ \\
\hline MUC17 & 7 & $\begin{array}{l}\text { NM_001040105.1: } \\
\text { c.8990T>C }\end{array}$ & $\begin{array}{l}\text { NP_001035194.1: } \\
\text { p.Met2997Thr }\end{array}$ & Substitution & Missense & rs193142121 & het & $9 \%$ \\
\hline$R P G R$ & $x$ & $\begin{array}{l}\text { NM_001034853.1: } \\
\text { c. } 2808 A>T\end{array}$ & $\begin{array}{l}\text { NP_001030025.1: } \\
\text { p.Glu936Asp }\end{array}$ & Substitution & Missense & rs187844918 & het & $20 \%$ \\
\hline GCNA & $x$ & $\begin{array}{c}\text { NM_052957.4: } \\
\text { c.883T >C }\end{array}$ & $\begin{array}{l}\text { NP_443189.1: } \\
\text { p.Ser295Pro }\end{array}$ & Substitution & Missense & & het & $12 \%$ \\
\hline
\end{tabular}

het, heterozygous; hom, homozygous.

aith 35\% tumor content in the sequenced sample. 
COLD SPRING HARBOR Molecular Case Studies
Therapeutic targets in recurrent pediatric papillary thyroid carcinoma compared to other inhibitors such as sunitinib, vandetanib, and axitinib (Verbeek et al. 2011). Access to cabozantinib may be possible through an early phase pediatric trial (NCT01709435), if this high-risk patient has further relapses.

\section{DISCUSSION}

Children with PTC have a high risk of recurrence, which may lead to multiple invasive surgeries and RAl. High cumulative lifetime doses of RAl carries a significant risk of late effects, including secondary malignancies and pulmonary fibrosis (Fard-Esfahani et al. 2014). Recurrent oncogenic drivers are frequently associated with childhood PTC, and tumor genomics may be leveraged to guide molecularly targeted therapy. We document a favorable response to an oral TRK inhibitor in an adolescent with multiply relapsed PTC.

These cases highlight the potential clinical utility of using tumor genomics to rationally guide targeted therapy. In the first case, a selective TRK inhibitor resulted in rapid and sustained radiographic improvement without toxicity. As the recognition of recurrent, actionable oncogenic drivers in pediatric thyroid carcinoma increases, there is an increasing need for development and access to clinically validated assays for rapid, cost-effective screening in relapsed, or refractory patients. Fusion panel assays may identify a proportion of recurrent actionable fusions, but this approach is not comprehensive and will likely miss other functionally equivalent fusions related to cryptic or noncanonical structural alterations. As the costs of WGS decrease and with improved turnaround times, clinically validated WGS assays may soon be available to facilitate comprehensive genomic analysis to identify potentially actionable oncogenic drivers in relapsed or refractory thyroid carcinoma.

Incorporation of transcriptome data in pediatric sequencing analyses may facilitate a deeper understanding of both oncogenic drivers, as well as treatment resistance, by delineating cellular pathway activation (Laskin et al. 2015). Interestingly, although these patients shared no genomic mutations, several genes such as PDGFRB, EGFR, GLI1, GLI2, and GLI3 are highly expressed in both samples. Given the limited sample size, it is unclear if this observation is by chance, or due to the difference between pediatric and adult thyroid carcinoma cohorts. The challenge in the pediatric setting is the availability of age-matched normal tissue and pediatric cancer reference compendia. With recent acceleration in the availability of genomic sequencing in childhood cancer, it is essential that data sharing initiatives are established and strengthened.

With increasing ability to identify oncogenic drivers in pediatric and adolescent oncology patients who fail upfront therapy, it is essential that we develop strategies to access molecularly guided clinical trials. Despite logistical challenges in pediatric oncology, molecularly guided phase II basket trials and novel trial designs are desperately needed.

\section{Conclusion}

Next-generation sequencing may frequently identify therapeutic targets in recurrent pediatric PTC. We demonstrate sustained efficacy of an oral TRK inhibitor in a child with multiply relapsed PTC and an oncogenic TRK fusion. The incorporation of genomic data in the routine care of relapsed pediatric PTC may help minimize treatment-related morbidity. Collaborative pediatric phase II studies of targeted therapy in PTC are needed to better define efficacy, toxicity and duration of response.

\section{METHODS}

Patients were enrolled in the Personalized Onco-Genomics (POG) project at BC Cancer in Vancouver, British Columbia. Tumor biopsies or surgical resections and blood samples were 
COLD SPRING HARBOR Molecular Case Studies
Therapeutic targets in recurrent pediatric papillary thyroid carcinoma

\begin{tabular}{lcrc}
\hline \multicolumn{1}{l}{ Table 3. Sequencing coverage } & \\
\hline & Tumor sample WGS & Blood sample WGS & $\begin{array}{c}\text { Tumor sample RNA-seq } \\
\text { coverage }\end{array}$ \\
\hline Patient 1 & $2,221,403,836$ reads (84.2-fold) & $1,156,446,710$ reads (43.8-fold) & $205,572,316$ reads $^{\text {a }}$ \\
Patient 2 & $2,030,407,160$ reads (82.9-fold) & $859,662,882$ read (33.8-fold) & $229,706,898$ reads \\
\hline
\end{tabular}

WGS, whole-genome sequencing.

a Read length is 75 bases.

collected for paired-end whole-genome and transcriptome sequencing. Whole-genome sequencing (WGS) depth is $>80 \times$ for tumor samples and $>30 \times$ for constitutional samples, and transcriptome sequencing depth is approximately $230 \mathrm{M}$ reads in total $(115 \mathrm{M}$ read pairs, Table 3). Somatic point mutations, small insertions or deletions (indels), copy-number alterations, structural variants were detected as described before (Jones et al. 2017). Gene expression levels were evaluated using public and in-house transcriptome sequencing data from the Illumina bodymap (www.illumina.com; ArrayExpress ID: E-MTAB-513) and The Cancer Genome Atlas (TCGA, https://tcga-data.nci.nih.gov/tcga/). Genes were linked to cancer pathways and drugs to identify potential therapeutic recommendations (Jones et al. 2017).

\section{ADDITIONAL INFORMATION}

\section{Data Deposition and Access}

As per the consent of POG, we do not share the sequencing data outside of the research group. The two structural variants were deposited to ClinVar (https://www.ncbi.nlm.nih. gov/clinvar/) and can be found under accession numbers SCV000693715 (the TPM3NTRK1 fusion) and SCV000693716 (the CCDC6-RET fusion).

\section{Ethics Statement}

Both patients and their guardians provided informed consent/assent for participation in the Pediatric Personalized Oncogenomics project, which is approved by the BC Children's and Women's Research Ethics Board.

\section{Acknowledgments}

We gratefully acknowledge the participation of our patients and families, the POG team, and the generous support of the BC Cancer Foundation and Genome British Columbia (project B20POG). We also acknowledge previous support from the National Cancer Institute ( $\mathrm{NCl})$ and the National Human Genome Research Institute (NHGRI) as part of The Cancer Genome Atlas project (U24 CA143866) and contributions toward equipment and infrastructure from Genome Canada and Genome BC (projects 202SEQ, 212SEQ, 12002), Canada Foundation for Innovation (projects 20070, 30198, 30981, 33408), and the BC Knowledge Development Fund. The results published here are in part based upon data generated by the following projects and obtained from dbGaP (http://www.ncbi.nlm.nih.gov/gap): The Cancer Genome Atlas managed by the NCl and NHGRI (http://cancergenome.nih.gov); Genotype-Tissue Expression (GTEx) Project, supported by the Common Fund of the Office of the Director of the National Institutes of Health (https://commonfund.nih.gov/GTEx); and the Therapeutically Applicable Research to Generate Effective Treatments (TARGET) initiative (dbGaP accession number phs000218.v16.p6) managed by the $\mathrm{NCl}$ (http://ocg.cancer. gov/programs/target). We would also like to acknowledge Loxo Oncology for providing larotrectinib treatment for our patient as part of its pediatric phase I/II trial (NCT02637687). 
COLD SPRING HARBOR Molecular Case Studies
Therapeutic targets in recurrent pediatric papillary thyroid carcinoma
Competing Interest Statement

The authors have declared no competing interest.

Received December 15, 2017; accepted in revised form February 3, 2018.

\section{Author Contributions}

R.R. participated in case series conception and prepared the first draft of the manuscript with revisions based on coauthor's feedback. S.R.R. participated in case series conception and manuscript preparation and revisions. Y.S. performed the bioinformatics analysis, created the data figures and participated in manuscript preparation and revisions. A.F.L., C.J., J.H., C.A., D.S.H., S.A., R.R., A.J.M., D.D., G.B., S.J.M.J., J.L., and M.A.M. participated in manuscript preparation and revisions. H.N. participated in manuscript revisions and provided imaging for the first case. R.J.D. was responsible for case series conception, manuscript preparation and revisions, and trainee supervision and oversaw completion of the project.

\section{REFERENCES}

Accardo G, Conzo G, Esposito D, Gambardella C, Mazzella M, Castaldo F, Di Donna C, Polistena A, Avenia N, Colantuoni V, et al. 2017. Genetics of medullary thyroid cancer: an overview. Int J Surg 41(Suppl 1): S2-S6.

Akshintala S, Children's Oncology Group. 2017. Phase 2 Study: Cabozantinib-S-Malate in Treating Young Patients with Recurrent, Refractory, or Newly Diagnosed Sarcomas, Wilms Tumor, or Other Rare Tumors. NCT02867592.

American Association for Cancer Research. 2016. TRK inhibitor shows early promise. Cancer Discov 6: OF4.

Beimfohr C, Klugbauer S, Demidchik EP, Lengfelder E, Rabes HM. 1999. NTRK1 re-arrangement in papillary thyroid carcinomas of children after the Chernobyl reactor accident. Int J Cancer 80: 842-847.

Cabanillas ME, Brose MS, Holland J, Ferguson KC, Sherman SI. 2014. A phase I study of cabozantinib (XL184) in patients with differentiated thyroid cancer. Thyroid 24: 1508-1514.

Food and Drug Administration. 2016. Highlights of prescribing information-CABOMETYX. FDA. https://www .accessdata.fda.gov/drugsatfda_docs/label/2016/208692s000lbl.pdf

Greco A, Miranda C, Pierotti MA. 2010. Rearrangements of NTRK1 gene in papillary thyroid carcinoma. Mol Cell Endocrinol 321: 44-49.

Fard-Esfahani A, Emami-Ardekani A, Fallahi B, Fard-Esfahani P, Beiki D, Hassanzadeh-Rad A, Eftekhari M. 2014. Adverse effects of radioactive iodine-131 treatment for differentiated thyroid carcinoma. Nucl Med Commun 35: 808-817.

Florenzano P, Guarda FJ, Jaimovich R, Droppelmann N, González H, Dominguez JM. 2016. Radioactive iodine administration is associated with persistent related symptoms in patients with differentiated thyroid cancer. Int J Endocrinol 2016: 2586512.

Jones MR, Lim H, Shen Y, Pleasance E, Ch'ng C, Reisle C, Leelakumari S, Zhao C, Yip S, Ho J, et al. 2017. Successful targeting of the NRG1 pathway indicates novel treatment strategy for metastatic cancer. Ann Oncol 28: 3092-3097.

Khotskaya YB, Holla VR, Farago AF, Mills Shaw KR, Meric-Bernstam F, Hong DS. 2017. Targeting TRK family proteins in cancer. Pharmacol Ther 173: 58-66.

Laetsch T, DuBois SG, Nagasubramanian R, Turpin B, Mascarenhas L, Federman N. 2017. A pediatric phase I study of larotrectinib, a highly selective inhibitor of the tropomyosin receptor kinase (TRK) family. Presented at ASCO June 52017.

Laskin J, Jones S, Aparicio S, Chia S, Ch'ng C, Deyell R, Eirew P, Fok A, Gelmon K, Ho C, et al. 2015. Lessons learned from the application of whole-genome analysis to the treatment of patients with advanced cancers. Cold Spring Harb Mol Case Stud 1: a000570.

Menicali E, Moretti S, Voce P, Romagnoli S, Avenia N, Puxeddu E. 2012. Intracellular signal transduction and modification of the tumor microenvironment induced by RET/PTCs in papillary thyroid carcinoma. Front Endocrinol (Lausanne) 3: 67.

Nikiforov YE, Nikiforova MN. 2011. Molecular genetics and diagnosis of thyroid cancer. Nat Rev Endocrinol 7: 569-580.

Parisi MT, Eslamy H, Mankoff D. 2016. Management of differentiated thyroid cancer in children: focus on the American thyroid association pediatric guidelines. Semin Nucl Med 46: 147-164

Prasad ML, Vyas M, Horne MJ, Virk RK, Morotti R, Liu Z, Tallini G, Nikiforova MN, Christison-Lagay ER, Udelsman R, et al. 2016. NTRK fusion oncogenes in pediatric papillary thyroid carcinoma in northeast United States. Cancer 122: 1097-1107.

Rubino C, de Vathaire F, Dottorini ME, Hall P, Schvartz C, Couette JE, Dondon MG, Abbas MT, Langlois C, Schlumberger M. 2003. Second primary malignancies in thyroid cancer patients. Br J Cancer 89: 16381644. 
COLD SPRING HARBOR

Molecular Case Studies
Therapeutic targets in recurrent pediatric papillary thyroid carcinoma

Santoro M, Carlomagno F. 2013. Central role of RET in thyroid cancer. Cold Spring Harb Perspect Biol 5: a009233.

The Cancer Genome Atlas Research Network. 2014. Integrated genomic characterization of papillary thyroid carcinoma. Cell 159: 676-690.

Verbeek HH, Alves MM, De Groot JW, Osinga J, Plukker JT, Links TP, Hofstra RM. 2011. The effects of four different tyrosine kinase inhibitors on medullary papillary thyroid cancer cells. J Clin Endocrinol Metab 96: e991-e995.

Xing M. 2013. Molecular pathogenesis and mechanisms of thyroid cancer. Nat Rev Cancer 13: 184-199. 


\section{COLD SPRING HARBOR Molecular Case Studies}

\section{Application of genomics to identify therapeutic targets in recurrent pediatric papillary thyroid carcinoma}

Rebecca Ronsley, S. Rod Rassekh, Yaoqing Shen, et al.

Cold Spring Harb Mol Case Stud 2018, 4: a002568

Access the most recent version at doi: $10.1101 / \mathrm{mcs} . a 002568$

References

License

Email Alerting Service
This article cites 19 articles, 3 of which can be accessed free at: http://molecularcasestudies.cshlp.org/content/4/2/a002568.full.html\#ref-list-1

This article is distributed under the terms of the Creative Commons Attribution-NonCommercial License, which permits reuse and redistribution, except for commercial purposes, provided that the original author and source are credited.

Receive free email alerts when new articles cite this article - sign up in the box at the top right corner of the article or click here. 